\title{
Implicit Variational Inclusions and Algorithms Involving $(A, \eta)$-monotone Operators in 2-uniformly Smooth Banach Spaces
}

\author{
N. K. Sahu \\ Dhirubhai Ambani Institute of Information and Communication Technology
}

Received: February 05, 2015 / Accepted: March 03, 2015 / Published: May 25, 2015.

\begin{abstract}
This paper deals with a new class of nonlinear set valued implicit variational inclusion problems involving $(A$, $\eta$ )-monotone mappings in 2-uniformly smooth Banach spaces. Semi-inner product structure has been used to study the $(A$, $\eta$ )-monotonicity. Using the generalized resolvent operator technique and the semi-inner product structure, the approximation solvability of the proposed problem is investigated. An iterative algorithm is constructed to approximate the solution of the problem. Convergence analysis of the proposed algorithm is investigated. Similar results are also investigated for variational inclusion problems involving $(H, \eta)$-monotone mappings.
\end{abstract}

Keywords: Semi-inner product space, Generalized resolvent operator, Variational inclusion, 2-uniformly smooth Banach space.

\section{Introduction}

Variational inclusion problem is a convenient framework for the unified study of many optimization related problems such as variational inequality problem, complementarity problem, fixed point problem, equilibrium problem etc. Due to its large applications in optimization related areas, variational inclusion problem has been well investigated. In the present scenario the development of efficient algorithms to solve variational inclusion problems is of more interest. To construct algorithms people use projection method and its variant forms, linear approximation, descent method and Newton's method. Resolvent operator technique is a generalization of projection method, and is largely used to solve variational inclusion problems (see [1], [4], [5], [6], [8], [11], [12] and [16]).

Monotonicity of the underlying operator plays a crucial role in solving variational inclusion problems.

Corresponding author: N. K. Sahu, Dhirubhai Ambani Institute of Information and Communication Technology. E-mail: nabin6582@gmail.com.
There are many generalizations of monotonicity such as maximal monotonicity, relaxed monotonicity, $H$-monotonicity, $A$-monotonicity, $(H, \eta)$-monotonicity and $(A, \eta)$-monotonicity. $(A, \eta)$-monotonicity was introduced in Verma [20], that generalizes the $A$-monotonicity concept ([21]) as well as $H$-monotonicity concept ([6]). Jin [9] investigated the approximation solvability of a new system of nonlinear set valued variational inclusion problems involving $(H, \eta)$-monotone operators. In 2008, Verma [19] solved a class of nonlinear set valued variational inclusions involving $(A, \eta)$-monotone operators in Hilbert space. Agarwal and Verma [2] solved a system of $(A, \eta)$-monotone variational inclusion problems in a Hilbert space using generalized hybrid algorithms. For some recent developments on variational inclusions one may refer to Moudafi [14], Verma [18], Ahmad et al. [3], Sahu et al. [17] and Noor et al. [15].

In this paper, our intent is to generalize the work of Verma [19]. Here we have solved a nonlinear set valued implicit variational inclusion problems in 2-uniformly smooth Banach space. In this paper we 
have used the semi-inner product structure to study those problems. Semi-inner product is a convenient and alternative approach to study different Hilbert space problems in Banach spaces. Semi-inner product helps us in a number of aspects for the smooth study of the problem. Hence we give some attention towards the theory of semi-inner product.

Definition 1. (Lumer [13]) Let $X$ be a vector space over the field $\mathrm{F}$ of real or complex numbers. A functional $[.,]:. X \times X \rightarrow F$ is called a semi-inner product if it satisfies the following:

(1) $[x+y, z]=[x, z]+[y, z], \forall x, y, z \in X$;

(2) $[\lambda x, y]=\lambda[x, y], \forall \lambda \in F$ and $x, y \in X$;

(3) $[x, x]>0$, for $x \neq 0$;

(4) $|[x, y]|^{2} \leq[x, x][y, y]$.

The pair $(X,[.,]$.$) is called a semi-inner product$ space.

A semi-inner product space is a normed linear space with the norm $\|x\|=[x, x]^{\frac{1}{2}}$. Every normed linear space can be made into a semi-inner product space in infinitely many different ways. Giles [7] had shown that if the underlying space $X$ is a uniformly convex smooth Banach space then it is possible to define a semi-inner product uniquely. For a detailed study and fundamental results on semi-inner product spaces, one may refer to Lumer [13], Giles [7] and Koehler [10].

Example 1. The real Banach space $L^{p}(\mathbb{R})$ for $1<p<\infty$ is a uniformly convex smooth Banach space with a unique semi-inner product defined by

$$
[f, g]=\frac{1}{\|g\|_{p}^{p-2}} \int_{\mathbb{R}} f(t)|g(t)|^{p-1} \operatorname{sgn}(g(t)) d t,
$$

$f, g \in L^{p}(\mathbb{R})$.

Example 2. The real sequence space $l^{p}$ for $1<p<\infty$ is a uniformly convex smooth space with a unique semi-inner product defined by

$$
[x, y]=\frac{1}{\|y\|_{p}^{p-2}} \sum_{i} x_{i} y_{i}\left|y_{i}\right|^{p-2}, x, y \in l^{p} .
$$

Definition 2. (Xu [23]) Let $X$ be a real Banach space. The modulus of smoothness of $X$ is defined as

$$
\begin{aligned}
& \rho_{X}(t)= \\
& \sup \left\{\frac{\|x+y\|+\|x-y\|}{2}-1:\|x\|=1,\|y\|=t, t>0\right\} .
\end{aligned}
$$

$X$ is said to be uniformly smooth if $\lim _{t \rightarrow 0} \frac{\rho_{X}(t)}{t}=0$.

$X$ is said to be $p$-uniformly smooth if there exists a positive real constant $c$ such that $\rho_{X}(t) \leq c t^{p}, \quad p>1$.

$X$ is said to be 2 -uniformly smooth if there exists a positive real constant $c$ such that $\rho_{X}(t) \leq c t^{2}$.

Lemma 1. (Xu [23]) Let $X$ be a smooth Banach space. Then the following statements are equivalent:

(1) $X$ is 2 -uniformly smooth.

(2) There is a constant $c>0$ such that for every $x, y \in X$, the following inequality holds

$$
\|x+y\|^{2} \leq\|x\|^{2}+2\left\langle y, f_{x}\right\rangle+c\|y\|^{2},
$$

where

$$
f_{x} \in J(x)
$$
and

$$
J(x)=\left\{x^{*} \in X^{*}:\left\langle x, x^{*}\right\rangle=\|x\|^{2} \text { and }\left\|x^{*}\right\|=\|x\|\right\}
$$

is the normalized duality mapping, where $X^{*}$ denotes the dual space of $X$ and $\left\langle x, x^{*}\right\rangle$ denotes the value of the functional $x^{*}$ at $x$, that is $x^{*}(x)$.

Remark 1. Every normed linear space is a semi-inner product space (see Lumer [13]). In fact by Hahn Banach theorem, for each $x \in X$, there exists at least one functional $f_{x} \in X^{*}$ such that $\left\langle x, f_{x}\right\rangle=\|x\|^{2}$. Given any such mapping $f$ from $X$ into $X^{*}$, it has been verified that $[y, x]=\left\langle y, f_{x}\right\rangle$ defines a semi-inner product. Hence we can write the inequality (1.1) as

$$
\|x+y\|^{2} \leq\|x\|^{2}+2[y, x]+c\|y\|^{2}, \forall x, y \in X .
$$

The constant $c$ is chosen with best possible minimum value. We call $c$, as the constant of smoothness of $X$.

Example 3. The functions space $L^{p}$ is 2 
-uniformly smooth for $p \geq 2$ and it is $p$-uniformly smooth for $1<p<2$.

If $2 \leq p<\infty$, then we have for all $x, y \in L^{p}$,

$\|x+y\|^{2} \leq\|x\|^{2}+2[y, x]+(p-1)\|y\|^{2}$.

Here the constant of smoothness is $p-1$.

\section{Preliminaries}

Let $X$ be a 2-uniformly smooth real Banach space with norm $\|$.$\| and semi-inner product [.,.].$ Let $2^{X}$ denotes the power set of $X$ and $C(X)$ denotes the collection of all closed subsets of $X$. Let us rewrite some of the definitions used in Sahu et al. [17] for completion of the paper, and write some new definitions.

Let $M: X \rightarrow 2^{X}$ be a set valued map. We denote both the mapping and its graph by $M$, that is $M=\{(x, y): y \in M(x)\}$. The domain of $M$ is defined by

$$
D(M)=\{x \in X: \exists y \in X:(x, y) \in M\} .
$$

The range of $M$ is defined by

$$
R(M)=\{y \in X: \exists x \in X:(x, y) \in M\} .
$$

The inverse $M^{-1}$ of $M$ is $\{(y, x):(x, y) \in M\}$.

For any two set valued mappings $N, M: X \rightarrow 2^{X}$ and any real number $\rho$, we define

$$
\begin{aligned}
& N+M=\{(x, y+z):(x, y) \in N,(x, z) \in M\}, \\
& \rho M=\{(x, \rho y):(x, y) \in M\} .
\end{aligned}
$$

For a map $A: X \rightarrow X$ and a set valued map $M: X \rightarrow 2^{X}$, we define

$$
A+M=\{(x, y+z): A x=y \text { and }(x, z) \in M\} .
$$

Definition 2.1. Let $\eta: X \times X \rightarrow X$ and $A, H: X \rightarrow X$ be single valued mappings. Let $M: X \rightarrow 2^{X}$ be a set valued map. Then the map $M$ is said to be:

(1) monotone if $[u-v, x-y] \geq 0$ for all $(x, u) \in M,(y, v) \in M$

(2) $\eta$-monotone if $[u-v, \eta(x, y)] \geq 0$ for all $(x, u) \in M,(y, v) \in M$;

(3) strictly $\eta$-monotone if $[u-v, \eta(x, y)] \geq 0$ for all $(x, u) \in M,(y, v) \in M$ except for $x=y$;

(4) $r$-strongly $\eta$-monotone if there exists a positive constant $r$ such that

$[u-v, \eta(x, y)] \geq r\|x-y\|^{2}$ for all $(x, u) \in M$, $(y, v) \in M$;

(5) $(m, \eta)$-relaxed monotone if there exists a constant $m>0$ such that

$[u-v, \eta(x, y)] \geq(-m)\|x-y\|^{2} \quad$ for $\quad$ all $(x, u) \in M,(y, v) \in M$;

(6) $\quad \eta$-firmly non expansive if $\|u-v\|^{2} \leq[u-v, \eta(x, y)]$ for all $(x, u) \in M$, $(y, v) \in M$;

(7) maximal monotone if $M$ is monotone and $(I+\rho M)(X)=X$ for all $\rho>0$, where $I$ is the identity mapping on $X$;

(8) maximal $\eta$-monotone if $M$ is $\eta$-monotone and $(I+\rho M)(X)=X$ for all $\rho>0$;

(9) $A$-monotone if $M$ is $m$-relaxed monotone and $(A+\rho M)(X)=X$ for all $\rho>0$;

(10) $(A, \eta)$-monotone if $M$ is $(m, \eta)$-relaxed monotone and $(A+\rho M)(X)=X$ for all $\rho>0$;

(11) $H$-monotone if $M$ is monotone and $(H+\rho M)(X)=X$ for all $\rho>0$;

(12) $(H, \eta)$-monotone if $M$ is $\eta$-monotone and $(H+\rho M)(X)=X$ for all $\rho>0$.

Lemma 2.1. Let $\eta: X \times X \rightarrow X$ be a single valued mapping, $A: X \rightarrow X$ be $r$-strongly $\eta$ -monotone mapping, and $M: X \rightarrow 2^{X}$ be an $(A, \eta)$-monotone mapping. Then the mapping $(A+\rho M)^{-1}: X \rightarrow X$ is single valued for $(r-\rho m)>0$.

Proof. For a given $u \in X$, assume that $x, y \in(A+\rho M)^{-1}(u)$. Then we have

$$
\frac{1}{\rho}(u-A(x)) \in M(x) ; \frac{1}{\rho}(u-A(y)) \in M(y) .
$$


Since $M$ is $(m, \eta)$-relaxed monotone, it follows that

$\frac{1}{\rho}[u-A(x)-(u-A(y)), \eta(x, y)] \geq(-m)\|x-y\|^{2}$ $\Rightarrow[A x-A y, \eta(x, y)] \leq(\rho m)\|x-y\|^{2}$.

Again $A$ is $r$-strongly $\eta$-monotone, that is $[A x-A y, \eta(x, y)] \geq r\|x-y\|^{2}, \forall x, y \in X$. Hence we have

$$
\begin{aligned}
& r\|x-y\|^{2} \leq[A x-A y, \eta(x, y)] \leq(\rho m)\|x-y\|^{2} \\
& \Rightarrow(r-\rho m)\|x-y\|^{2} \leq[A x-A y, \eta(x, y)] \leq 0 .
\end{aligned}
$$

This is possible only when $x=y$, since $r-\rho m$ is positive. Hence one can conclude that the operator $(A+\rho M)^{-1}$ is single valued.

Now we can define the generalized resolvent operator associated with $(A, \eta)$-monotone mapping.

Definition 2.2. Let $\eta: X \times X \rightarrow X$ be a single valued mapping, $A: X \rightarrow X$ be an $r$ -strongly $\eta$-monotone mapping, and $M: X \rightarrow 2^{X}$ be an $(A, \eta)$-monotone mapping. Then the generalized resolvent operator $J_{\rho, M}^{A, \eta}: X \rightarrow X$ is defined by

$$
J_{\rho, M}^{A, \eta}(x)=(A+\rho M)^{-1}(x) \text { for all } x \in X,
$$

where $\rho$ is a positive constant.

Definition 2.3. A mapping $\eta: X \times X \rightarrow X$ is said to be $\tau$-Lipschitz continuous if there exists a constant $\tau>0$ such that $\|\eta(x, y)\| \leq \tau\|x-y\|$ for all $x, y \in X$.

Lemma 2.2. Let $\eta: X \times X \rightarrow X$ be a $\tau$ -Lipschitz continuous map, $A: X \rightarrow X$ be an $r$ -strongly $\eta$-monotone mapping and $M: X \rightarrow 2^{X}$ be an $(A, \eta)$-monotone mapping. Then the generalized resolvent operator $J_{\rho, M}^{A, \eta}: X \rightarrow X$ is $\frac{\tau}{r-\rho m}$-Lipschitz continuous for $0<\rho<\frac{r}{m}$.

Proof. For any $x, y \in X$, we have

$$
\begin{aligned}
& J_{\rho, M}^{A, \eta}(x)=(A+\rho M)^{-1}(x) \\
& J_{\rho, M}^{A, \eta}(y)=(A+\rho M)^{-1}(y) .
\end{aligned}
$$

This implies that

$$
\begin{aligned}
& \frac{1}{\rho}\left\{x-A J_{\rho, M}^{A, \eta}(x)\right\} \in M\left(J_{\rho, M}^{A, \eta}(x)\right) \\
& \frac{1}{\rho}\left\{y-A J_{\rho, M}^{A, \eta}(y)\right\} \in M\left(J_{\rho, M}^{A, \eta}(y)\right) .
\end{aligned}
$$

Since $M$ is $(m, \eta)$-relaxed monotone, we have

$$
\begin{aligned}
& \frac{1}{\rho}\left[x-A J_{\rho, M}^{A, \eta}(x)-\left\{y-A J_{\rho, M}^{A, \eta}(y)\right\},\right. \\
& \left.\eta\left(J_{\rho, M}^{A, \eta}(x), J_{\rho, M}^{A, \eta}(y)\right)\right] \\
& =\frac{1}{\rho}\left[x-y-\left\{A J_{\rho, M}^{A, \eta}(x)-A J_{\rho, M}^{A, \eta}(y)\right\},\right. \\
& \left.\eta\left(J_{\rho, M}^{A, \eta}(x), J_{\rho, M}^{A, \eta}(y)\right)\right] \\
& \geq(-m)\left\|J_{\rho, M}^{A, \eta}(x)-J_{\rho, M}^{A, \eta}(y)\right\|^{2} .
\end{aligned}
$$

Using the $r$-strongly $\eta$-monotonicity of the operator $A$ in the above inequality, we get

$$
\begin{aligned}
& \|x-y\|\left\|\eta\left(J_{\rho, M}^{A, \eta}(x), J_{\rho, M}^{A, \eta}(y)\right)\right\| \\
& \geq\left[x-y, \eta\left(J_{\rho, M}^{A, \eta}(x), J_{\rho, M}^{A, \eta}(y)\right)\right] \\
& =\left[x-y-\left\{A J_{\rho, M}^{A, \eta}(x)-A J_{\rho, M}^{A, \eta}(y)\right\},\right. \\
& \left.\eta\left(J_{\rho, M}^{A, \eta}(x), J_{\rho, M}^{A, \eta}(y)\right)\right] \\
& +\left[A J_{\rho, M}^{A, \eta}(x)-A J_{\rho, M}^{A, \eta}(y),\right. \\
& \left.\eta\left(J_{\rho, M}^{A, \eta}(x), J_{\rho, M}^{A, \eta}(y)\right)\right] \\
& \geq-\rho m\left\|J_{\rho, M}^{A, \eta}(x)-J_{\rho, M}^{A, \eta}(y)\right\|^{2} \\
& +r\left\|J_{\rho, M}^{A, \eta}(x)-J_{\rho, M}^{A, \eta}(y)\right\|^{2} \\
& =(r-\rho m)\left\|J_{\rho, M}^{A, \eta}(x)-J_{\rho, M}^{A, \eta}(y)\right\|^{2} .
\end{aligned}
$$

Applying the $\tau$-Lipschitz continuity of $\eta$ in the above inequality, we get

$$
\begin{gathered}
\\
\|x-y\| \tau\left\|J_{\rho, M}^{A, \eta}(x)-J_{\rho, M}^{A, \eta}(y)\right\| \\
\geq\|x-y\|\left\|\eta\left(J_{\rho, M}^{A, \eta}(x), J_{\rho, M}^{A, \eta}(y)\right)\right\| \\
\geq(r-\rho m)\left\|J_{\rho, M}^{A, \eta}(x)-J_{\rho, M}^{A, \eta}(y)\right\|^{2} .
\end{gathered}
$$


This implies that

$$
\begin{aligned}
& \left\|J_{\rho, M}^{A, \eta}(x)-J_{\rho, M}^{A, \eta}(y)\right\| \leq \frac{\tau}{r-\rho m}\|x-y\|, \\
& \text { where } 0<\rho<\frac{r}{m} .
\end{aligned}
$$

It is not difficult to prove similar results as Lemma 2.1 and Lemma 2.2 for $(H, \eta)$-monotone operators. We state those results without proof.

Lemma 2.3. Let $\eta: X \times X \rightarrow X$ be a single valued mapping, $H: X \rightarrow X$ be $r$-strongly $\eta$ -monotone mapping, and $M: X \rightarrow 2^{X}$ be an $(H$, $\eta)$-monotone mapping. Then the mapping $(H+\rho M)^{-1}$ is single valued.

Definition 2.4. Let $\eta: X \times X \rightarrow X$ be a single valued mapping, $H: X \rightarrow X$ be $r$-strongly $\eta$ -monotone mapping, and $M: X \rightarrow 2^{X}$ be an $(H$, $\eta)$-monotone mapping. Then the generalized resolvent operator $J_{\rho, M}^{H, \eta}: X \rightarrow X$ is defined by

$$
J_{\rho, M}^{H, \eta}(x)=(H+\rho M)^{-1}(x) \text { for all } x \in X,
$$

where $\rho$ is a positive constant.

Lemma 2.4. Let $\eta: X \times X \rightarrow X$ be a $\tau$ -Lipschitz continuous map, $H: X \rightarrow X$ be an $r$ -strongly $\eta$-monotone mapping and $M: X \rightarrow 2^{X}$ be an $(H, \eta)$-monotone mapping. Then the generalized resolvent operator $J_{\rho, M}^{H, \eta}: X \rightarrow X$ is $\frac{\tau}{r}$-Lipschitz continuous.

In the following proposition, we discuss another important characteristic of the generalized resolvent operator associated with $(A, \eta)$-monotone operator.

Proposition 2.1. Let $\eta: X \times X \rightarrow X$ be a $\tau$ -Lipschitz continuous map, $A: X \rightarrow X$ be an $r$ -strongly $\eta$-monotone mapping and $M: X \rightarrow 2^{X}$ be an $(A, \eta)$-monotone mapping. Then the generalized resolvent operator $J_{\rho, M}^{A, \eta}: X \rightarrow X$ is $\left(\frac{\tau^{2}}{r-\rho m}, \eta\right)$ -firmly nonexpansive.

Proof. For any $x, y \in X$, we have

$$
\begin{aligned}
& \frac{1}{\rho}\left\{x-A J_{\rho, M}^{A, \eta}(x)\right\} \in M\left(J_{\rho, M}^{A, \eta}(x)\right) \\
& \frac{1}{\rho}\left\{y-A J_{\rho, M}^{A, \eta}(y)\right\} \in M\left(J_{\rho, M}^{A, \eta}(y)\right) .
\end{aligned}
$$

As in the proof of Lemma 2.2, we have

$$
\begin{aligned}
& {\left[x-y, \eta\left(J_{\rho, M}^{A, \eta}(x), J_{\rho, M}^{A, \eta}(y)\right)\right]} \\
& \geq(r-\rho m)\left\|J_{\rho, M}^{A, \eta}(x)-J_{\rho, M}^{A, \eta}(y)\right\|^{2} .
\end{aligned}
$$

Since $\eta$ is $\tau$-Lipschitz continuous, we get

$$
\begin{aligned}
\left\|\eta\left(J_{\rho, M}^{A, \eta}(x), J_{\rho, M}^{A, \eta}(y)\right)\right\| & \leq \tau\left\|J_{\rho, M}^{A, \eta}(x)-J_{\rho, M}^{A, \eta}(y)\right\| \\
\Rightarrow\left\|J_{\rho, M}^{A, \eta}(x)-J_{\rho, M}^{A, \eta}(y)\right\| & \geq \frac{1}{\tau}\left\|\eta\left(J_{\rho, M}^{A, \eta}(x), J_{\rho, M}^{A, \eta}(y)\right)\right\| .
\end{aligned}
$$

Using (2.4) in (2.3), we get

$$
\begin{aligned}
& {\left[x-y, \eta\left(J_{\rho, M}^{A, \eta}(x), J_{\rho, M}^{A, \eta}(y)\right)\right]} \\
& \geq \frac{r-\rho m}{\tau^{2}}\left\|\eta\left(J_{\rho, M}^{A, \eta}(x), J_{\rho, M}^{A, \eta}(y)\right)\right\|^{2} \\
& \Rightarrow\left\|\eta\left(J_{\rho, M}^{A, \eta}(x), J_{\rho, M}^{A, \eta}(y)\right)\right\|^{2} \\
& \leq \frac{\tau^{2}}{r-\rho m}\left[x-y, \eta\left(J_{\rho, M}^{A, \eta}(x), J_{\rho, M}^{A, \eta}(y)\right)\right] .
\end{aligned}
$$

Housdorff pseudo-metric $D: 2^{X} \times 2^{X} \rightarrow R \cup\{+\infty\}$ is defined as

$$
D(A, B)=\max \left\{\sup _{x \in A} \inf _{y \in B}\|x-y\|, \sup _{x \in B} \inf _{y \in A}\|x-y\|\right\}
$$

for that any two subsets $A$ and $B$ of $X$. When the domain $2^{X}$ is restricted to the closed bounded subsets of $X$, then $D$ is the Housdorff metric.

Definition 2.5. A set valued map $M: X \rightarrow 2^{X}$ is said to be $D$-Lipschitz continuous if there exists a constant $v>0$ such that

$$
D(M(x), M(y)) \leq v\|x-y\| \text { for all } x, y \in X .
$$

\section{Implicit Set Valued Variational Inclusion and Its Solvability}

In this section we state the problem that we are intended to solve and discuss its various forms. We discuss the solvability method and construct an 
iterative algorithm using the generalized resolvent operator technique to approximate the solution. We also explore the convergence analysis of the proposed algorithm.

Let $X$ be a 2 -uniformly smooth real Banach space and $K$ be a nonempty closed convex subset of $X$. Let $S, \eta: X \times X \rightarrow X, A, g: X \rightarrow X$ be nonlinear mappings. Let $U: X \rightarrow 2^{X}$ be a set valued mapping and $M: X \rightarrow 2^{X}$ be an $(A, \eta)$ -monotone mapping. We consider the following set valued, nonlinear implicit variational inclusion problem:

Find $x \in X, u \in U(x)$ such that

$$
0 \in S(x, u)+M(g(x)) .
$$

\section{Special cases:}

When $g=I$, the problem (3.5) reduces to the following non implicit variational inclusion problem.

Find $x \in X, u \in U(x)$ such that

$$
0 \in S(x, u)+M(x) .
$$

The above problem was solved by Verma [19] in a Hilbert space.

When $M$ is $(H, \eta)$-monotone, the non implicit problem (3.6) was solved by Fang et al. [6] in Hilbert space.

When $X$ is a 2 -uniformly smooth real Banach space and $M: X \rightarrow 2^{X}$ be a set valued mapping. If $S, T: X \rightarrow X$ are any single valued mappings and $g: X \rightarrow X$ be any mapping such that Range $(g) \cap \operatorname{Dom}(M)$ is nonempty. Then the following implicit variational inclusion problem:

For a given element $x \in X$, find an element $a \in X$ such that

$$
x \in S(a)-T(a)+M(g(a))
$$

was solved by Sahu et al. [17].

When $g=I$, the Identity, the above problem (3.7) was studied by Verma [18] in a Hilbert space.

When $x=0$ and $T=0$, the above problem was solved by Verma [22] in a Hilbert space.
First we discuss an equivalent formulation of the proposed problem (3.5). Using the equivalent formulation and generalized resolvent operator, we construct an iterative algorithm to approximate the solution of the problem.

Theorem 3.1. For a given $x \in X, u \in U(x)$, the pair $(x, u)$ is a solution of the variational inclusion problem (3.5) if and only if

$$
g(x)=J_{\rho, M}^{A, \eta}(A(g(x))-\rho S(x, u)),
$$

where $\rho$ is a positive real constant.

Proof. Let $(x, u)$ be a solution of the variational inclusion problem (3.5). That is

$$
\begin{aligned}
& 0 \in S(x, u)+M(g(x)) \\
& \Rightarrow A(g(x)) \in A(g(x))+\rho S(x, u)+\rho M(g(x)) \\
& \Rightarrow A(g(x))-\rho S(x, u) \in(A+\rho M)(g(x)) \\
& \Rightarrow g(x)=J_{\rho, M}^{A, \eta}(A(g(x))-\rho S(x, u)) .
\end{aligned}
$$

Conversely, let $g(x)=J_{\rho, M}^{A, \eta}(A(g(x))-\rho S(x, u))$. This implies that

$$
\begin{aligned}
& A(g(x))-\rho S(x, u) \in(A+\rho M)(g(x)) \\
& \Rightarrow A(g(x))-\rho S(x, u) \in A(g(x))+\rho M(g(x)) \\
& \Rightarrow 0 \in S(x, u)+M(g(x)) .
\end{aligned}
$$

Based on the above Theorem 3.1, we construct a general iterative method to approximate the solution of (3.5).

\section{Algorithm 3.1.}

Step 1. Choose initial approximation $x_{0} \in X$ and $u_{0} \in U(x)$

Step 2. Construct the sequence $\left\{x_{k}\right\}$ such that

$$
\begin{aligned}
& x_{k+1}=(1-\alpha) x_{k}+\alpha\left\{\left(x_{k}-g\left(x_{k}\right)\right)+\right. \\
& \left.J_{\rho, M}^{A, \eta}\left(A g\left(x_{k}\right)-\rho S\left(x_{k}, u_{k}\right)\right)\right\},
\end{aligned}
$$

where $\alpha \in(0,1]$ is a constant.

Step 3. Choose $u_{k+1} \in U\left(x_{k+1}\right)$ such that

$$
\left\|u_{k+1}-u_{k}\right\| \leq\left(1+(1+k)^{-1}\right) D\left(U\left(x_{k+1}\right), U\left(x_{k}\right)\right),
$$


where $D(.,$.$) is the Housdorff pseudo-metric on$ $2^{X}$.

Step 4. If $x_{k+1}$ and $u_{k+1}$ satisfy (3.9) with a sufficient degree of accuracy, then stop. Else set $k=k+1$ and go to Step 2 .

Theorem 3.2. Let $\eta: X \times X \rightarrow X$ be $\tau$ -Lipschitz continuous, $A: X \rightarrow X$ be $r$-strongly $\eta$-monotone and $\beta$-Lipschitz continuous. Let $M: X \rightarrow 2^{X} \quad$ be $\quad(A, \eta)$-monotone and $U: X \rightarrow C(X)$ be $D-\gamma$-Lipschitz continuous. Let $g: X \rightarrow X$ be $t$-strongly monotone and $s$ -Lipschitz continuous. Let $S: X \times X \rightarrow X$ be a nonlinear mapping such that for any given element $(a, b) \in X \times X, S(., b)$ is $\mu$-strongly monotone with respect to $A g$ and $\alpha$-Lipschitz continuous, and $S(a,$.$) is \xi$-Lipschitz continuous. Moreover, if there exists a constant $0<\rho<\frac{r}{m}$ such that

$$
\begin{aligned}
& \sqrt{1-2 t+c s^{2}}(r-\rho m)+ \\
& \tau \sqrt{\beta^{2} s^{2}+c \rho^{2} \alpha^{2}-2 \rho \mu}+\tau \rho \xi \gamma<r-\rho m,
\end{aligned}
$$

then the problem (3.5) has a solution (x,u), and the sequences $x_{k}$ and $u_{k}$, generated by Algorithm 3.1, converges strongly to the solution $x$ and $u$, respectively.

Proof. Using the iterative scheme (3.9), we have

$$
\begin{gathered}
\left\|x_{k+1}-x_{k}\right\| \\
=\|(1-\alpha) x_{k}+\alpha\left\{\left(x_{k}-g\left(x_{k}\right)\right)\right. \\
\left.+J_{\rho, M}^{A, \eta}\left(A g\left(x_{k}\right)-\rho S\left(x_{k}, u_{k}\right)\right)\right\} \\
-(1-\alpha) x_{k-1}+\alpha\left\{\left(x_{k-1}-g\left(x_{k-1}\right)\right)\right. \\
\left.+J_{\rho, M}^{A, \eta}\left(A g\left(x_{k-1}\right)-\rho S\left(x_{k-1}, u_{k-1}\right)\right)\right\} \| \\
\leq(1-\alpha)\left\|x_{k}-x_{k-1}\right\|+\alpha \| x_{k} \\
-x_{k-1}-\left(g\left(x_{k}\right)-g\left(x_{k-1}\right)\right) \| \\
+\alpha \| J_{\rho, M}^{A, \eta}\left(\operatorname{Ag}\left(x_{k}\right)-\rho S\left(x_{k}, u_{k}\right)\right) \\
-J_{\rho, M}^{A, \eta}\left(A g\left(x_{k-1}\right)-\rho S\left(x_{k-1}, u_{k-1}\right)\right) \| \\
\leq(1-\alpha)\left\|x_{k}-x_{k-1}\right\|+\alpha \| x_{k} \\
-x_{k-1}-\left(g\left(x_{k}\right)-g\left(x_{k-1}\right)\right) \|
\end{gathered}
$$

$$
\begin{aligned}
& +\frac{\alpha \tau}{r-\rho m} \| A g\left(x_{k}\right)-\rho S\left(x_{k}, u_{k}\right) \\
- & \left\{A g\left(x_{k-1}\right)-\rho S\left(x_{k-1}, u_{k-1}\right)\right\} \| \\
= & (1-\alpha)\left\|x_{k}-x_{k-1}\right\|+ \\
\alpha & \left\|x_{k}-x_{k-1}-\left(g\left(x_{k}\right)-g\left(x_{k-1}\right)\right)\right\| \\
& +\frac{\alpha \tau}{r-\rho m} \| A g\left(x_{k}\right)-A g\left(x_{k-1}\right) \\
- & \left\{\rho S\left(x_{k}, u_{k}\right)-\rho S\left(x_{k-1}, u_{k-1}\right)\right\} \| \\
\leq & (1-\alpha)\left\|x_{k}-x_{k-1}\right\| \\
+ & \alpha\left\|x_{k}-x_{k-1}-\left(g\left(x_{k}\right)-g\left(x_{k-1}\right)\right)\right\| \\
& +\frac{\alpha \tau}{r-\rho m} \| A g\left(x_{k}\right)-A g\left(x_{k-1}\right) \\
& -\rho\left\{S\left(x_{k}, u_{k}\right)-S\left(x_{k-1}, u_{k}\right)\right\} \| \\
+ & \frac{\alpha \tau \rho}{r-\rho m}\left\|S\left(x_{k-1}, u_{k}\right)-S\left(x_{k-1}, u_{k-1}\right)\right\|
\end{aligned}
$$

Using the argument in Remark 1.1, and since $g$ is $t$-strongly monotone and $s$-Lipschitz continuous, we have

$$
\begin{aligned}
& \left\|x_{k}-x_{k-1}-\left(g\left(x_{k}\right)-g\left(x_{k-1}\right)\right)\right\|^{2} \\
& \leq\left\|x_{k}-x_{k-1}\right\|^{2}-2\left[g\left(x_{k}\right)-g\left(x_{k-1}\right), x_{k}-x_{k-1}\right] \\
& +c\left\|g\left(x_{k}\right)-g\left(x_{k-1}\right)\right\|^{2} \\
& \leq\left\|x_{k}-x_{k-1}\right\|^{2}-2 t\left\|x_{k}-x_{k-1}\right\|^{2}+c s^{2}\left\|x_{k}-x_{k-1}\right\|^{2} \\
& =\left(1-2 t+c s^{2}\right)\left\|x_{k}-x_{k-1}\right\|^{2} . \\
& \quad \Rightarrow\left\|x_{k}-x_{k-1}-\left(g\left(x_{k}\right)-g\left(x_{k-1}\right)\right)\right\| \\
& \quad \leq \sqrt{1-2 t+c s^{2}}\left\|x_{k}-x_{k-1}\right\|
\end{aligned}
$$

Using the similar argument, and since $A$ is $\beta$ -Lipschitz continuous and $S(., b)$ is $\mu$ strongly monotone with respect to $A g$, we get 


$$
\begin{aligned}
& \| A g\left(x_{k}\right)-A g\left(x_{k-1}\right)- \\
& \rho\left\{S\left(x_{k}, u_{k}\right)-S\left(x_{k-1}, u_{k}\right)\right\} \|^{2} \\
& \leq\left\|A g\left(x_{k}\right)-A g\left(x_{k-1}\right)\right\|^{2}- \\
& 2 \rho\left[S\left(x_{k}, u_{k}\right)-S\left(x_{k-1}, u_{k}\right), A g\left(x_{k}\right)-A g\left(x_{k-1}\right)\right] \\
& +c \rho^{2}\left\|S\left(x_{k}, u_{k}\right)-S\left(x_{k-1}, u_{k}\right)\right\|^{2} \\
& \leq \beta^{2}\left\|g\left(x_{k}\right)-g\left(x_{k-1}\right)\right\|^{2}-2 \rho \mu \| x_{k} \\
& -x_{k-1}\left\|^{2}+c \rho^{2} \alpha^{2}\right\| x_{k}-x_{k-1} \|^{2} \\
& \leq \beta^{2} S^{2}\left\|x_{k}-x_{k-1}\right\|^{2}-2 \rho \mu \| x_{k} \\
& -x_{k-1}\left\|\left.\right|^{2}+c \rho^{2} \alpha^{2}\right\| x_{k}-x_{k-1} \|^{2} \\
& =\left(\beta^{2} S^{2}+c \rho^{2} \alpha^{2}-2 \rho \mu\right)\left\|x_{k}-x_{k-1}\right\|^{2} . \\
& \quad \Rightarrow \| A g\left(x_{k}\right)-A g\left(x_{k-1}\right)- \\
& \quad \rho\left\{S\left(x_{k}, u_{k}\right)-S\left(x_{k-1}, u_{k}\right)\right\} \| \\
& \leq \sqrt{\beta^{2} s^{2}+c \rho^{2} \alpha^{2}-2 \rho \mu}\left\|x_{k}-x_{k-1}\right\| .
\end{aligned}
$$

Again using (3.10), and since $S(a,$.$) is \xi$ -Lipschitz continuous and $U$ is $D-\gamma$-Lipschitz continuous, we have

$$
\begin{gathered}
\left\|S\left(x_{k-1}, u_{k}\right)-S\left(x_{k-1}, u_{k-1}\right)\right\| \leq \xi\left\|u_{k}-u_{k-1}\right\| \\
\leq \xi\left(1+k^{-1}\right) D\left(U\left(x_{k}\right), U\left(x_{k-1}\right)\right) \\
\leq \xi \gamma\left(1+k^{-1}\right)\left\|x_{k}-x_{k-1}\right\| .
\end{gathered}
$$

Placing (3.13), (3.14) and (3.15) in (3.12), we get

$$
\begin{aligned}
& \left\|x_{k+1}-x_{k}\right\| \\
& \leq(1-\alpha)\left\|x_{k}-x_{k-1}\right\|+\alpha \sqrt{1-2 t+c s^{2}}\left\|x_{k}-x_{k-1}\right\| \\
& +\frac{\alpha \tau}{r-\rho m} \sqrt{\beta^{2} s^{2}+c \rho^{2} \alpha^{2}-2 \rho \mu} \| x_{k} \\
& -x_{k-1}\left\|+\frac{\alpha \tau \rho}{r-\rho m} \xi \gamma\left(1+k^{-1}\right)\right\| x_{k}-x_{k-1} \| . \\
& \Rightarrow\left\|x_{k+1}-x_{k}\right\| \\
& \leq\left(1-\alpha+\alpha \sqrt{1-2 t+c s^{2}}+\right. \\
& \frac{\alpha \tau}{r-\rho m} \sqrt{\beta^{2} s^{2}+c \rho^{2} \alpha^{2}-2 \rho \mu} \\
& \left.+\frac{\alpha \tau \rho}{r-\rho m} \xi \gamma\left(1+k^{-1}\right)\right)
\end{aligned}
$$

$\left\|x_{k}-x_{k-1}\right\| \leq\left(1-\alpha+\alpha \theta_{k}\right)\left\|x_{k}-x_{k-1}\right\|$,

where

$$
\begin{aligned}
& \theta_{k}=\sqrt{1-2 t+c s^{2}}+ \\
& \frac{\tau}{r-\rho m} \sqrt{\beta^{2} s^{2}+c \rho^{2} \alpha^{2}-2 \rho \mu} \\
& +\frac{\tau \rho}{r-\rho m} \xi \gamma\left(1+k^{-1}\right) .
\end{aligned}
$$

If

$$
\begin{aligned}
& \theta=\sqrt{1-2 t+c s^{2}}+ \\
& \frac{\tau}{r-\rho m} \sqrt{\beta^{2} s^{2}+c \rho^{2} \alpha^{2}-2 \rho \mu}+\frac{\tau \rho}{r-\rho m} \xi \gamma,
\end{aligned}
$$

then we can see that $\theta_{k} \rightarrow \theta$ as $k \rightarrow \infty$.

Because of the assumption (3.11), we have $0<\theta<1$. Therefore by inequality (3.16), and since $0<\alpha \leq 1$, one can conclude that $\left\{x_{k}\right\}$ is a Cauchy sequence. Since $X$ is complete, there exists $x \in X$ such that $x_{k} \rightarrow x$ as $k \rightarrow \infty$.

Next we prove that $u_{k} \rightarrow u \in U(x)$ as $k \rightarrow \infty$. Inequality (3.10) guarantees that $\left\{u_{k}\right\}$ is also a Cauchy sequence in $X$. Therefore there exists $u \in X$ such that $u_{k} \rightarrow u$ as $k \rightarrow \infty$. Moreover,

$$
d(u, U(x))=\inf \{\|u-t\|: t \in U(x)\}
$$

$$
\begin{gathered}
\quad \leq\left\|u-u_{k}\right\|+d\left(u_{k}, U(x)\right) \\
\leq\left\|u-u_{k}\right\|+D\left(U\left(x_{k}\right), U(x)\right) \\
\leq\left\|u-u_{k}\right\|+\gamma\left\|x_{k}-x\right\| . \quad(3.17)
\end{gathered}
$$

$d(u, U(x)) \rightarrow 0$ as $k \rightarrow \infty$ because the right hand side of (3.17) goes to 0 as $k \rightarrow \infty$. Therefore, since $U(x)$ is closed, we have $u \in U(x)$.

The continuity property of $g, J_{\rho, M}^{A, \eta}, A$ and $S$ makes sure that

$$
g(x)=J_{\rho, M}^{A, \eta}(A(g(x))-\rho S(x, u)) .
$$

Then from Theorem 3.1, we conclude that the 
duplet $(x, u)$ is a solution of the variational inclusion problem (3.5).

\subsection{Implicit Variational Inclusions Involving} $(H, \eta)$-monotone Operators

Here we consider the same problem as the problem (3.5), but we impose the $(H, \eta)$-monotonicity on the set valued operator $M$ in stead of $(A, \eta)$ -monotonicity. We state the problem in the following manner:

Let $X$ be a 2 -uniformly smooth real Banach space and $K$ be a nonempty closed convex subset of $X$. Let $S, \eta: X \times X \rightarrow X, H, g: X \rightarrow X$ be nonlinear mappings. Let $U: X \rightarrow 2^{X}$ be a set valued mapping and $M: X \rightarrow 2^{X}$ be an $(H, \eta)$ -monotone mapping. We consider the following set valued, nonlinear implicit variational inclusion problem:

Find $x \in X, u \in U(x)$ such that

$$
0 \in S(x, u)+M(g(x)) .
$$

As usual, we establish the fixed point type equivalent formulation of the proposed problem in the following manner. The proof is parallel to the proof of Theorem 3.1 .

Theorem 3.3. For a given $x \in X, u \in U(x)$, the pair $(x, u)$ is a solution of the variational inclusion problem (3.18) if and only if

$$
g(x)=J_{\rho, M}^{H, \eta}(H(g(x))-\rho S(x, u)),
$$

where $\rho$ is a positive real constant.

As a result of the above Theorem 3.3, we construct an iterative algorithm to solve our proposed problem (3.18).

\section{Algorithm 3.2.}

Step 1. Choose initial approximation $x_{0} \in X$ and $u_{0} \in U(x)$

Step 2. Construct the sequence $\left\{x_{k}\right\}$ such that

$$
\begin{aligned}
& x_{k+1}=(1-\alpha) x_{k}+\alpha\left\{\left(x_{k}-g\left(x_{k}\right)\right)\right. \\
& \left.+J_{\rho, M}^{H, \eta}\left(H g\left(x_{k}\right)-\rho S\left(x_{k}, u_{k}\right)\right)\right\},
\end{aligned}
$$

where $\alpha \in(0,1]$ is a constant.

Step 3. Choose $u_{k+1} \in U\left(x_{k+1}\right)$ such that

$$
\left\|u_{k+1}-u_{k}\right\| \leq\left(1+(1+k)^{-1}\right) D\left(U\left(x_{k+1}\right), U\left(x_{k}\right)\right),
$$

where $D(.,$.$) is the Housdorff pseudo-metric on$ $2^{X}$.

Step 4. If $x_{k+1}$ and $u_{k+1}$ satisfy (3.20) with a sufficient degree of accuracy, then stop. Else set $k=k+1$ and go to Step 2 .

Finally, we state the convergence theorem of the above iterative algorithm to the solution of the Problem (3.18).

Theorem 3.4. Let $\eta: X \times X \rightarrow X$ be $\tau$ -Lipschitz continuous, $H: X \rightarrow X$ be $r$-strongly $\eta$-monotone and $\beta$-Lipschitz continuous. Let $M: X \rightarrow 2^{X} \quad$ be $(H, \eta) \quad$-monotone and $U: X \rightarrow C(X)$ be $D-\gamma$-Lipschitz continuous. Let $g: X \rightarrow X$ be $t$-strongly monotone and $s$ -Lipschitz continuous. Let $S: X \times X \rightarrow X$ be a nonlinear mapping such that for any given element $(a, b) \in X \times X, S(., b)$ is $\mu$-strongly monotone with respect to $H g$ and $\alpha$-Lipschitz continuous, and $S(a,$.$) is \xi$-Lipschitz continuous. Moreover, if

$$
\begin{aligned}
& \sqrt{1-2 t+c s^{2}} r+ \\
& \tau \sqrt{\beta^{2} s^{2}+c \rho^{2} \alpha^{2}-2 \rho \mu}+\tau \rho \xi \gamma<r,
\end{aligned}
$$

then the problem (3.18) has a solution $(\mathrm{x}, \mathrm{u})$, and the sequences $x_{k}$ and $u_{k}$, generated by Algorithm 3.2, converges strongly to the solution $x$ and $u$, respectively.

Proof. The proof is similar to the proof of Theorem 3.2, but we have to use Lemma 2.4 in stead of Lemma 2.2 .

\section{References}

[1] S. Adly, Perturbed algorithm and sensitivity analysis for a general class of variational inclusions, J. Math. Anal. Appl. 201 (1996), 609-630.

[2] R. P. Agarwal and R. U. Verma, General system of $(A, \eta)$-maximal relaxed monotone variational inclusion 
problems based on generalized hybrid algorithms, Commun. Nonlinear Sci. Numer. Simulat. 15 (2010) 238-251.

[3] R. Ahmad, M. Akram and J. C. Yao, Generalized monotone mapping with an application for solving a variational inclusion problem, J. Optim. Theory Appl. 157(2) (2013), 324-346.

[4] X. P. Ding, Perturbed proximal point for generalized quasi-variational inclusions, J. Math. Anal. Appl. 210 (1997), 88-101.

[5] X. P. Ding and C. L. Luo, Perturbed proximal point algorithms for generalized quasi-variational like inclusions, J. Comput. Appl. Math. 210 (2000), 153-165.

[6] Y. P. Fang and N. J. Huang, $H$-monotone operator and resolvent operator technique for variational inclusions, Appl. Math. Comput. 145 (2003), 795-803.

[7] J. R. Giles, Classes of semi-inner product spaces, Trans. Amer. Math. Soc. 129 (1967), 436-446.

[8] N. J. Huang, A new class of generalized set valued implicit variational inclusions in Banach spaces with an application, Comput. Math. Appl. 41 (2001), 937-943.

[9] M. M. Jin, Iterative algorithm for a new system of nonlinear set-valued variational inclusions involving $(H, \eta)$-monotone mappings, J. Inequal. Pure Appl. Math. 7(2) (2006), Article 72.

[10] D. O. Koehler, A note on some operator theory in certain semi-inner product spaces, Proc. Amer. Math. Soc. 30(2) (1971), 363-366.

[11] H. Y. Lan, J. H. Kim and Y. J. Cho, On a new system of nonlinear $A$-monotone multivalued variational inclusions, J. Math. Anal. Appl. 327 (2007), 481-493.

[12] C. H. Lee, Q. H. Ansari and J. C. Yao, A perturbed algorithm for strongly nonlinear variational like inclusions, Bull. Aust. Math. Soc. 62 (2000), 417-426.

[13] G. Lumer, Semi-inner product spaces, Trans. Amer. Math.
Soc. 100 (1961), 29-43.

[14] A. Moudafi, Split monotone variational inclusions, $J$. Optim. Theory Appl. 150(2) (2011), 275-283.

[15] M. A. Noor, K. I. Noor and R. Kamal, General variational inclusions involving difference of operators, J. Inequal. Appl. 2014(1) (2014), Article ID 98.

[16] J. Peng and D. Zhu, A new system of generalized mixed quasi-variational inclusions with $(H, \eta)$-monotone operators, J. Math. Anal. Appl. 327 (2007), 175-187.

[17] N. K. Sahu, R. N. Mohapatra, C. Nahak and S. Nanda, Approximation solvability of a class of $A$-monotone implicit variational inclusion problems in semi-inner product spaces, Appl. Math. Comput. 236 (2014), 109-117.

[18] R. U. Verma, General nonlinear variational inclusion problems involving $A$-monotone mappings, Appl. Math. Lett., , (2006) 960-963.

[19] R. U. Verma, Approximation solvability of a class of nonlinear set-valued variational inclusions involving

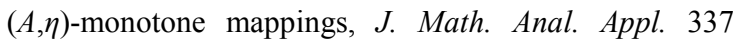
(2008), 969-975.

[20] R. U. Verma, Sensitivity analysis for generalized strongly monotone variational inclusions based on $(A, \eta)$-resolvent operator technique, Appl. Math. Lett. 19 (2006), 1409-1413.

[21] R. U. Verma, $A$-monotonicity and its role in nonlinear variational inclusions, J. Optim. Theory Appl. 129(3) (2006), 457-467.

[22] R. U. Verma, General class of implicit variational inclusions and graph convergence on $A$-maximal relaxed monotonicity, J. Optim. Theory Appl., 155 (1), (2012), 196-214.

[23] H. K. Xu, Inequalities in Banach spaces with applications, Nonlinear Analysis: Theory, Methods and Applications 16(12) (1991), 1127-1138. 